\title{
Frequency Dispersion Compensation Through Variable Window Utilization in Time Reversal Techniques for Electromagnetic Waves
}

DOI:

10.1109/TAP.2016.2565724

\section{Document Version}

Accepted author manuscript

Link to publication record in Manchester Research Explorer

Citation for published version (APA):

Abduljabbar, A., Yavuz, M. E., Costen, F., Himeno, R., \& Yokota, H. (2016). Frequency Dispersion Compensation Through Variable Window Utilization in Time Reversal Techniques for Electromagnetic Waves. IEEE Transactions on Antennas and Propagation, 64(8). https://doi.org/10.1109/TAP.2016.2565724

\section{Published in:}

IEEE Transactions on Antennas and Propagation

\section{Citing this paper}

Please note that where the full-text provided on Manchester Research Explorer is the Author Accepted Manuscript or Proof version this may differ from the final Published version. If citing, it is advised that you check and use the publisher's definitive version.

\section{General rights}

Copyright and moral rights for the publications made accessible in the Research Explorer are retained by the authors and/or other copyright owners and it is a condition of accessing publications that users recognise and abide by the legal requirements associated with these rights.

\section{Takedown policy}

If you believe that this document breaches copyright please refer to the University of Manchester's Takedown Procedures [http://man.ac.uk/04Y6Bo] or contact uml.scholarlycommunications@manchester.ac.uk providing relevant details, so we can investigate your claim.

\section{OPEN ACCESS}




\section{Frequency Dispersion Compensation Through Variable Window Utilization in Time Reversal Techniques for Electromagnetic Waves}

Ammar M. Abduljabbar, Student Member, IEEE, Mehmet E. Yavuz, Fumie Costen, Senior Member, IEEE, Ryutaro

Himeno, Hideo Yokota

\begin{abstract}
In microwave imaging applications, propagating signals undergo additional attenuation in dispersive and lossy media when compared to non-dispersive and lossless media. In this paper, we introduce a threshold approach and Short-Time Fourier transform (STFT) based inverse filters to compensate for such additional attenuation in time reversal (TR) based imaging algorithms. The method introduced here is utilized to reduce the unwanted noise amplification in the received signals during the compensation stage. Additionally, optimum settings for window type and length in the STFT method are obtained through a scanning operation in the propagation medium. Different window types and lengths are studied to achieve best focusing resolution in TR applications. While utilizing large number of windows with short spatial lengths provides improved TR focusing performance, it also increases the overall cost and complexity of the imaging system. The threshold method introduced here achieves improved TR focusing performance without increasing the cost by utilizing a lower number of inverse filters.
\end{abstract}

Index Terms-Time reversal (TR), short time Fourier transform (STFT), dispersive media, attenuation compensation, finite difference time domain (FDTD).

\section{INTRODUCTION}

Localization of targets behind structures or within obstructed media is of great interest in many microwave imaging applications such as detection of landmines [1], [2], explosives [3] or tumors [4]. A recent method that has been introduced to tackle the detection and localization problem is the time-reversal (TR) technique [5]. TR simply states that for every wave propagating away from a source following a certain path, there is a corresponding time-reversed wave signal that can trace the same path back to the original point of the source. This path can be traced back through the medium even across variation in permittivity and permeability. The first step of locating an object within a medium using the TR technique is to send a pulse through the medium and receive the reflected signals from the object. TR technique uses a set of antennas, called a TR Array (TRA), to receive, record and time-reverse the reflected signal from the object. All time-reversed signals at each TRA antenna are sent back to the medium to focus around the object location at the time of refocus. One important property of TR is that it achieves super-resolution by utilizing the multipath propagation in the

A. M. Abduljabbar, and F. Costen are with the School of Electrical and Electronic Engineering, The University of Manchester, U.K. (email: fumie.costen@manchester.ac.uk)

M. E. Yavuz, Hillsboro, Oregon, USA. (email: yavuz.5@osu.edu)

R. Himeno is Advanced Center for Computing and Communication, RIKEN, Saitama, Japan

H. Yokota and F. Costen are with the Image Processing Research Team, Center for Advanced Photonics, RIKEN, Saitama, Japan.

Color versions of the figures in this paper are available online at http://ieeexplore.iee.org. medium as discussed in [6], [7], [8] and allows interference cancellation [9] and detection [10]. On the other hand, as with other microwave imaging techniques, TR suffers from dispersion and losses in the propagating medium [11], [12]. While the additional phase shift undergone due to dispersion can be compensated by the TR process itself, attenuation affects the signals in both the forward and backward propagation. This attenuation ultimately degrades the focusing resolution of the imaging process. The earliest works on compensating such attenuation involved a uniform absorption model over the entire bandwidth for acoustic propagation in skull [13], multipaths in ocean communication [14] and at a single frequency with the so called decomposition of the time-reversal operator method [15]. A more recent compensation technique using the STFT method takes the frequency dependency account for the first time and tries to improve the resolution focusing of the TR technique in dispersive and lossy media [11]. Although this method proved to be a better candidate for compensation, the inverse filters utilized in the method also amplify unwanted oscillations and perturbations (noise) in the high frequency components of the received signals' spectra, which affect the performance of TR technique. In our proposed method, we apply a threshold approach to the inverse filters in order to reduce the effects of this oscillation and perturbation. We refer to these oscillations and perturbations as noise. Furthermore, we apply different types and lengths of windows to select the optimum settings for the STFT method while [11] used a single window type with a fixed length. The details of the proposed compensation method is presented in Section II. Section III-A and Section III-B provide the radio environment setting and the results and analysis for the numerical experiments. Section IV shows the conclusions and the future plan.

\section{COMPENSATION OF DISPERSIVE ATTENUATION IN} HUMAN TISSUES

A dispersive and lossy medium acts like a filter for signals propagating through it. An inverse filter is needed to compensate for the losses of the propagating signals in such media. The real part of the permittivity $\epsilon$ of the propagation medium causes a phase shift to the traveling waves during the forward propagation of the TR technique. The phase shift is corrected in the backward propagation due to the fact that the signals in the backward propagation are phase-conjugated coherently along all the bandwidth [16]. The imaginary part of $\epsilon$ causes signal attenuation.

We propose a method to compensate this attenuation as shown in Fig. 1. Our method starts by time-windowing the signal $\mathbf{x}[n]$ observed at each TRA antenna, setting

$$
\boldsymbol{X}_{i}[n]=\mathbf{x}[n] \boldsymbol{W}_{i}[n] \quad \text { for } \quad i=1, \ldots, M
$$

where $n$ is the time step $(0 \leq n \leq N), N$ is the total time steps of $\mathbf{x}[n], \boldsymbol{W}_{i}[n]$ is the $i$ th window, $\boldsymbol{X}_{i}[n]$ is the $i$ th windowed time domain signal and $M$ is the total number of windows.

$M$ is selected based on $N$ as $M=\left\lceil\frac{2 N}{l}\right\rceil$ where \lceil\rceil represents the ceiling function. The window function [17] 


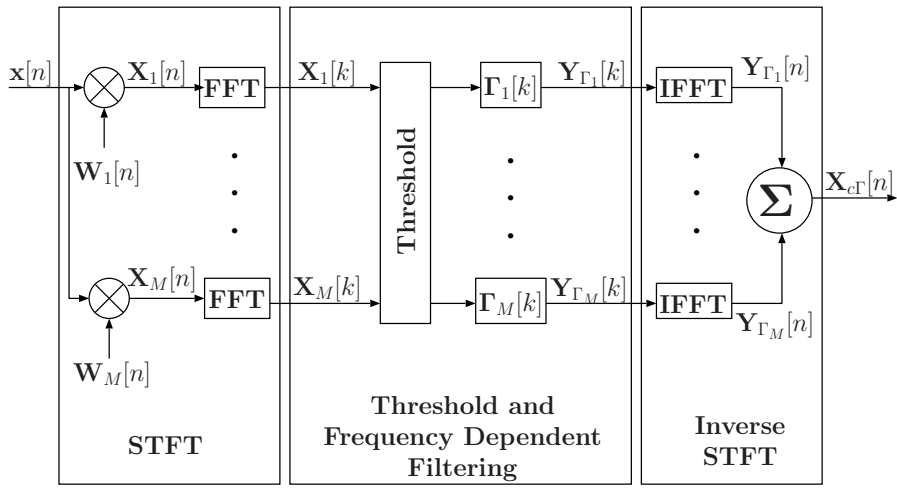

Fig. 1. The flowchart of the compensation method with variable windows utilized for improved imaging performance.

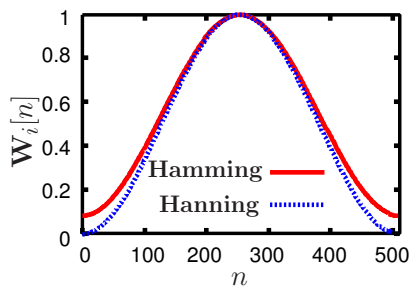

(a) Hanning and Hamming.

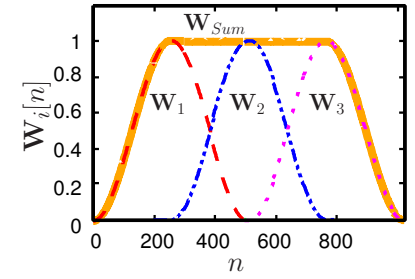

(b) Three Hanning windows with overlapping factor of 0.5 .
Fig. 2. Different window types with $l=512$ and $\boldsymbol{W}_{S u m}=\boldsymbol{W}_{1}+\boldsymbol{W}_{2}+$ $\boldsymbol{W}_{3}$.

is either a Hanning or Hamming window whose $\boldsymbol{W}_{i}[n]$ is expressed as

$$
\boldsymbol{W}_{i}[n]=\frac{1}{2}\left(1-\cos \left(\frac{2 \pi}{l}\left(n-\varsigma_{i}\right)\right)\right) \quad \varsigma_{i} \leq n \leq \varsigma_{i}+l
$$

for the Hanning window and

$$
\boldsymbol{W}_{i}[n]=0.54-0.46 \cos \left(\frac{2 \pi}{l}\left(n-\varsigma_{i}\right)\right) \quad \varsigma_{i} \leq n \leq \varsigma_{i}+l
$$

for the Hamming window where $\varsigma_{i}=\left[\frac{l(i-1)}{2}\right]$, with [] representing the integer value and $l$ is the window's length. $\boldsymbol{W}_{i}[n]=0$ beyond $\varsigma_{i} \leq n \leq \varsigma_{i}+l$ limits.

Fig. 2(a) shows examples of Hanning and Hamming windows with $l=512$ time steps. Fig. 2(b) shows three Hanning windows and their summation with $l=512$ time steps and an overlapping factor of 0.5 . Overlapping guarantees that the original signal can be retrieved if all the windows are summed together. The Hanning window can avoid the an abrupt change in the time-gated signals by approaching zero near the edges of the window. In [11] a Hamming window was used with a fixed $l$ of 256 time steps, which is not optimized for the STFT method. The Hamming window causes an abrupt change in the time-gated signals near the edges of the window. Therefore, we used Hanning and Hamming windows with $32 \leq l \leq 512$ to optimize $l$ for our compensation method. Another drawback of the compensation method in [11] is that the inverse filters amplify the noise in the observed signals. We propose a threshold approach to reduce the noise amplification that was inevitable in [11].
$\boldsymbol{X}_{i}[n]$ is converted to the frequency domain $\boldsymbol{X}_{i}[k]$ where $k$ represents the frequency. In our threshold approach we set the threshold to $e^{-1}$ [18] of the peak of the spectrum of the original signal $\mathbf{x}[k]$. The threshold value is set to $e^{-1}$ to obtain the optimum signal to noise ratio (SNR) in different types of human tissues (propagation media) including fat (least dispersive medium) and muscle (most dispersive medium). Therefore, we defined the highest frequency of interest as the frequency at which the spectrum is $e^{-1}$ relative to the spectrum maximum [18]. The inverse filters utilized in [11] not only amplify the signal of interest, but also they amplify the noise reducing the SNR and in return this lowers the accuracy of the localization. When the threshold is set to $e^{-1}$ the noise amplification is reduced and the localization is improved. The inverse filters amplify the noise (i.e. SNR is reduced) when the threshold value is set to less than $e^{-1}$. This reduces the amplitude of $\boldsymbol{X}_{i}[k]$ relative to noise. This SNR reduction decreases the accuracy of the localization and (7) is not satisfied. When the threshold value is set to higher than $e^{-1}$ the noise is not amplified. However the inverse filters are not applied to the high frequency components of $\boldsymbol{X}_{i}[k]$ within the frequency range of interest causing the SNR to drop again. This reduces the accuracy of the localization and (7) is not satisfied. We apply the compensation filter at those frequencies $k$ where the spectrum $\boldsymbol{X}_{i}[k]$ is higher than the threshold. $\boldsymbol{X}_{i}[n]$ has traveled different distances to reach the TRA antennas and each frequency component has gone through different attenuation. Therefore, $\boldsymbol{X}_{i}[k]$ has to be compensated with a different space and frequency dependent filter, $\boldsymbol{\Gamma}_{i}[k]$.

$\boldsymbol{\Gamma}_{i}[k]$ is produced for the $i$ th window by comparing observed signals in the dispersive medium with a corresponding nondispersive medium. More details about space and frequency dependent filter are presented in [11]. The filtered signal in the frequency domain is

$$
\boldsymbol{Y}_{\Gamma_{i}}[k]=\boldsymbol{X}_{i}[k] \boldsymbol{\Gamma}_{i}[k] \quad \text { for } \quad i=1, \ldots, M .
$$

This frequency dependent filter acts like an inverse filter with respect to the attenuation of the dispersive medium. Due to the finite length of the filters, additional phase shifts may have to be introduced. These phase shifts do not affect the focusing around the scatterer location because all the observed signals go through the same filters [11]. We apply the inverse STFT to each filtered signal by converting $\boldsymbol{Y}_{\Gamma_{i}}[k]$ to a time domain signal $\boldsymbol{Y}_{\Gamma_{i}}[n]$ and sum all $\boldsymbol{Y}_{\Gamma_{i}}[n]$ back together to obtain the compensated signal

$$
\boldsymbol{X}_{c \Gamma}[n]=\sum_{i=1}^{M} \boldsymbol{Y}_{\Gamma_{i}}[n] .
$$

Varying $l$ from 32 to 512 the optimum $l$ is identified for the Hanning and Hamming windows by the following procedure: Step 1) Find $\left(i_{m}, j_{m}\right)$ which satisfy

$$
\left|E_{z}\left(i_{m}, j_{m}\right)\right|=\max _{i, j}\left|E_{z}(i, j)\right| \triangleq \mathcal{Q}
$$

where $E_{z}$ is the electric field component in the $z$ direction, $\mathcal{Q}$ represents the maximum value of the $\left|E_{z}\right|$ field in the entire FDTD space at the time of 


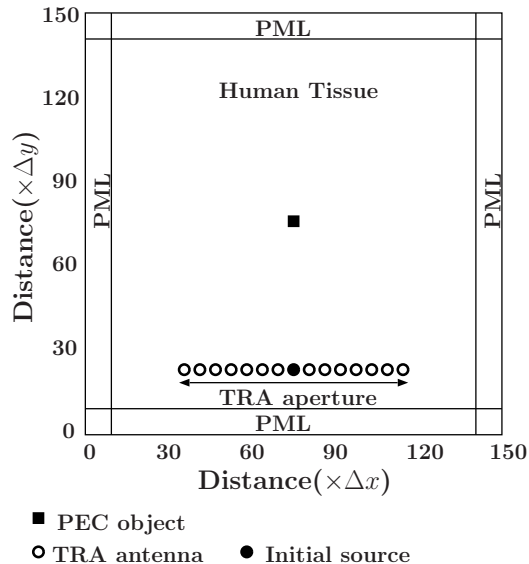

Fig. 3. Simulation setup.

refocusing and $\left(i_{m}, j_{m}\right)$ is the location of $\mathcal{Q}$ in the FDTD space.

Step 2) Select $l$ with which $\left(i_{m}, j_{m}\right)$ satisfy

$$
\left(i_{m}-i_{p}\right)^{2}+\left(j_{m}-j_{p}\right)^{2} \leq 2
$$

which is regarded as a successful localization where $\left(i_{p}, j_{p}\right)$ represents the center of the scatterer location. Step 3) Calculate $S_{l}=\sum_{i, j} \frac{\left|E_{z}(i, j)\right|}{\mathcal{Q}}$ where $(i, j)$ satisfy $\left(i-i_{p}\right)^{2}+\left(j-j_{p}\right)^{2} \leq 2$.

Step 4) Choose $l$ which gives the minimum $S_{l}$.

\section{NUMERICAL EXPERIMENTS}

The purpose of these experiments is to compare the results of our compensation method and the original STFT method of [11]. Our proposed threshold approach reduces the amplification of the unwanted noise, resulting in more accurate spatial focusing.

\section{A. Radio Environment Setting}

The simulation setup is depicted in Fig. 3. All the simulations use the $\mathrm{TM}_{z}$ polarized 2D-FDTD method. The FDTD space is $150 \times 150$, uniformly sampled with a spatial step of $\Delta s \triangleq \Delta x=\Delta y=1 \mathrm{~mm}$. The time step $\Delta t$ is set to $2.357 \mathrm{ps}$. Complex Frequency-Shifted Perfectly Matched Layer (CFSPML) absorbing boundary conditions [19] are used with a 9cell layer. The TRA is composed of 15 transceivers antennas, equally spaced from each other and parallel to the $x$-axis. The TRA has the aperture of $70 \Delta s$. The 1st TRA antenna is located at $(40 \Delta x, 25 \Delta y)$ and the 15 th is located at $(110 \Delta x, 25 \Delta y)$. A $3 \times 3$ Perfect Electric Conductor (PEC) scatterer is located in the medium and centered at $(75 \Delta x, 75 \Delta y) \triangleq\left(i_{p}, j_{p}\right)$. The human tissues are modeled using the one-pole Debye media [20] where the relative permittivity is $\epsilon_{r}=\epsilon_{\infty}+\frac{\epsilon_{\mathrm{S}}-\epsilon_{\infty}}{1+\jmath \omega \tau_{\mathrm{D}}}-\jmath \frac{\sigma}{\omega \epsilon_{0}}$ where $\epsilon_{\infty}$ is the infinite frequency relative permittivity, $\epsilon_{\mathrm{S}}$ is the static frequency relative permittivity, $\omega$ is the angular frequency, $\tau_{\mathrm{D}}$ is the relaxation time, $\sigma$ is the conductivity and $\epsilon_{0}$ is the free space permittivity.
TABLE I

PARAmeters For THE ONE POLE Debye Media

\begin{tabular}{lllll}
\hline Medium & $\sigma[\mathrm{S} / \mathrm{m}]$ & $\epsilon_{\mathrm{S}}$ & $\epsilon_{\infty}$ & $\tau_{\mathrm{D}}[\mathrm{ps}]$ \\
\hline \hline Fat & 0.037 & 05.530 & 03.998 & 23.630 \\
Bone & 0.104 & 14.169 & 07.363 & 34.100 \\
Muscle & 0.747 & 56.931 & 28.001 & 18.672 \\
Test medium & 0.0005 & 7.0 & 5.5 & 4851.0 \\
\hline \hline
\end{tabular}

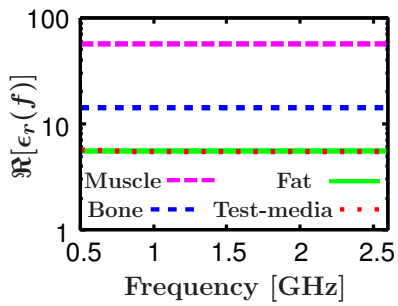

(a) $\Re\left[\epsilon_{r}\right]$.

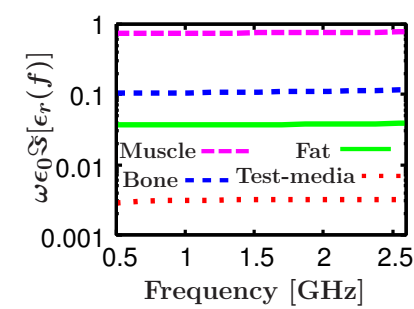

(b) $\omega \epsilon_{0} \Im\left[\epsilon_{r}\right]$.
Fig. 4. $\Re\left[\epsilon_{r}\right]$ and $\omega \epsilon_{0} \Im\left[\epsilon_{r}\right]$ for different tissues.

The 8th (center) antenna of the TRA transmits a first derivative Gaussian pulse into the medium. As this propagates through the dispersive medium, it is scattered by the PEC object and then recorded by the TRA antennas. The recorded signals on each TRA antenna are then compensated, time reversed, and transmitted back to the medium. The timereversed signals automatically focus on the position of the scatterer. We apply our compensation method to the cases where the FDTD space is homogeneously filled either with fat, bone or muscle. Table I shows the media parameters for the one-pole Debye relaxation model [21] for the human tissues as well as a test medium set to have similar characteristics to those of the two-species Lorentz medium in [11]. Among the tissues considered here, fat is the least dispersive, muscle is the most dispersive and bone is in-between these two. Fig. 4(a) and (b) show the real and imaginary parts of the relative permittivity respectively for all the media in Table I. The test medium in [11] is less dispersive than the human tissues. The phase shift of the fat is almost the same as the test medium but the attenuation of the fat is higher than the test medium. Muscle has the highest attenuation and phase shift followed by bone, fat and the test medium.

\section{B. Numerical Results}

We measure the distance between the location of the maximum value of $\left|E_{z}\right|\left(i_{m}, j_{m}\right)$ at the time of refocusing and the center of the scatterer location of $\left(i_{p}, j_{p}\right)$ for the proposed method and the method in [8] and calculate the accuracy $A$ as

$$
A=\frac{s_{o}}{s_{n}}
$$

where $s_{o}$ and $s_{n}$ are the distances between $\left(i_{m}, j_{m}\right)$ and $\left(i_{p}, j_{p}\right)$ for the method in [11] and our compensation method respectively. Both approaches satisfied (7) in the fat medium. The proposed method is $(A=) 1.14$ times more accurate than the method in [11]. In the case of propagation in bone and muscle, the proposed approach satisfied (7) while the method in [11] did not. The inverse filter in [11] amplify the 
TABLE II

OPTIMIZED $l$ IN STEP 4

\begin{tabular}{lll}
\hline Medium & Hanning & Hamming \\
\hline \hline Fat & 482 & 482 \\
Bone & 422 & 422 \\
Muscle & 242 & 212 \\
\hline
\end{tabular}

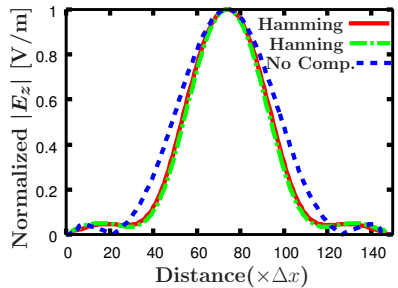

(a) Bone case.

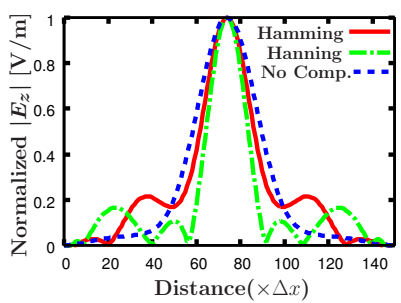

(b) Muscle case.
Fig. 5. The cross-section of the normalized $\left|E_{z}\right|$ distribution at $y=75 \Delta y$ in human tissues with our compensation method using either Hanning or Hamming and without them.

noise (reducing the SNR) in highly dispersive tissues such as bone and muscle. The amplification of the noise reduces the amplitude of $X_{c \Gamma}$ relative to noise which reduces the accuracy of the localization and dislocates $\left(i_{m}, j_{m}\right)$ far from $\left(i_{p}, j_{p}\right)$. In our approach, the noise amplification is reduced as the inverse filters are not applied on the high frequency part of the spectrum.

Table II shows the value of $l$ which achieves the sharpest focusing in fat, bone and muscle using either Hanning or Hamming window based on our four-step procedure. In the muscle medium, the Hanning window requires less $M\left(=\left\lceil\frac{2 N}{l}\right\rceil\right)$ than the Hamming windows, leading to reduction in the cost of our compensation method.

Fig. 5(a) and (b) show cross-sections of the normalized $\left|E_{z}\right|$ at $y=75 \Delta y$ in bone and muscle respectively. The spatial resolution of the focusing with our compensation method is higher than without it. We notice that the resolution of the focusing is higher with Hanning than with Hamming. Fig. 6(a) shows the observed signal at the 1st antenna of the TRA for the bone case with and without our compensation method. Fig. 6(b) shows the compensated signal using the method in [11]. There are oscillations in our reconstructed signal in Fig. 6(a). These oscillations are caused by the noise amplification of the filters. However, without our threshold approach the noise level becomes so large as shown in Fig. 6(b) that the reconstructed signal is strongly distorted and the focusing is lost. Fig. 7(a) and (b) shows $\left|E_{z}\right|$ in the $x y$ plane in bone with and without our compensation method. The resolution of the spatial focusing becomes higher with our compensation method. Fig. 8 shows the cross-range of Fig. 7 at scatterer location $(y=75 \Delta y) . R_{c}=\left(x_{3}-x_{2}\right) \Delta x$ and $R_{n}=\left(x_{4}-x_{1}\right) \Delta x$ represent the resolution of the spatial focusing with and without our compensation method respectively where $x_{1}, x_{2}, x_{3}$ and $x_{4}$ are spatial points on the $x$ axis and $\left|E_{z}\left(x_{1}\right)\right|=\left|E_{z}\left(x_{2}\right)\right|=\left|E_{z}\left(x_{3}\right)\right|=\left|E_{z}\left(x_{4}\right)\right|=0.5$ where $E_{z}$ is normalized and 0.5 is the cross-range at half maximum to calculate the resolution of the spatial focusing. The

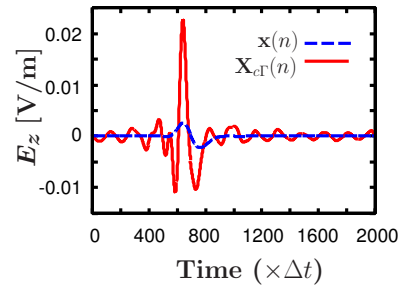

(a) $\mathbf{x}[n]$ and $\boldsymbol{X}_{c \Gamma}[n]$ using our method.

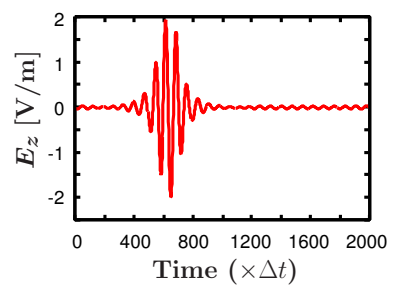

(b) $\boldsymbol{X}_{c \Gamma}[n]$ using the method in [11].
Fig. 6. The observed signal $\mathbf{x}[n]$ for the bone case at the 1 st antenna of the TRA without compensation, with our compensation $\left(\boldsymbol{X}_{c \Gamma}[n]\right)$ and with [11] compensation method.

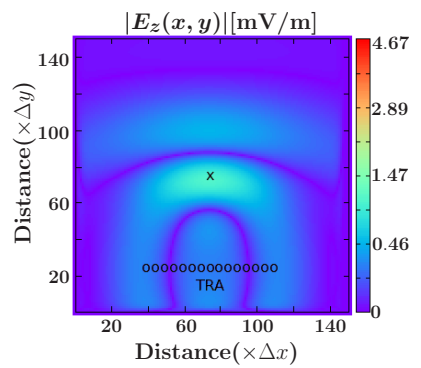

(a) no compensation

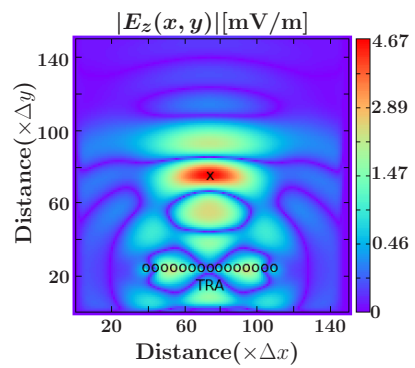

(b) with compensation
Fig. 7. The spatial distribution of the $\left|E_{z}\right|$ field component in the $x y$-plane for a dispersive medium composed of bone tissue (with and without our compensation). The scatterer is represented by a small " $\times$ ". $\Delta x=\Delta y=1 \mathrm{~mm}$.

spatial resolution of the proposed method is $\left(\frac{R_{n}}{R_{c}}=\right) 1.25$ times higher than the one without any compensation in the propagation medium of bone.

\section{CONCLUSION}

The wave equation invariance of the time reversal technique is broken in human tissues due to their dispersive characteristics. In this paper, we have introduced a threshold and inverse filter based technique to improve a previously developed compensation scheme utilizing the STFT method. Thanks

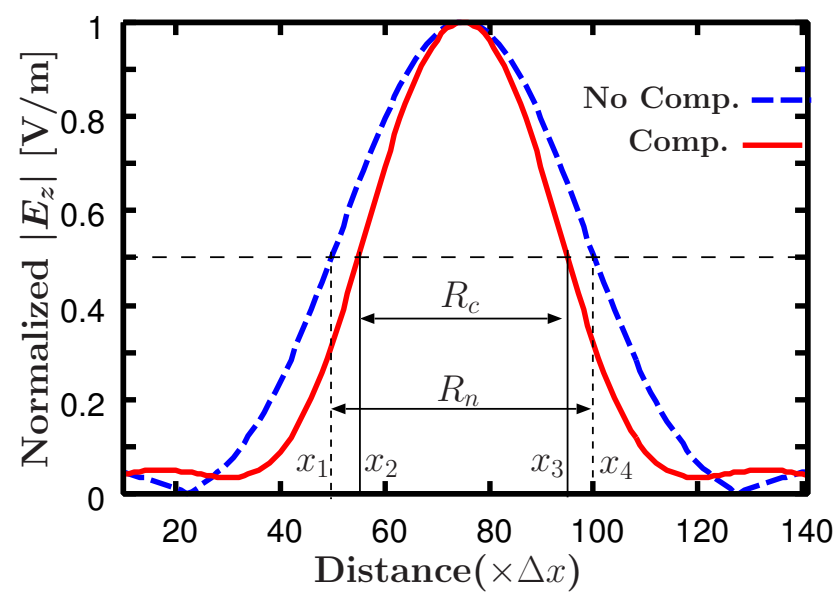

Fig. 8. The cross-section of the normalized $\left|E_{z}\right|$ distribution at $y=75 \Delta y$ for bone with the proposed compensation method (Comp.) and without any compensation method (No Comp.). 
to the threshold approach, the reduction of unwanted noise amplification is achieved. Additionally, through the optimal selection of window type and lengths used at the STFT stage, adaptive compensation is achieved depending on the scenario. We have shown that the choice of Hanning window improved the localization of the scatterers as compared to the Hamming window as it inherently avoids the discontinuity at the edges of the window. We obtained improved results in terms of localization and spatial distribution of $E_{z}$ around the object location. While STFT is robust for tackling the attenuation problem in dispersive media, several other methods such as the S-transform or wavelet transforms can also be investigated in this framework which are some proposed future works for the researchers in the field.

\section{REFERENCES}

[1] P. D. Gader, M. Mystkowski, and Y. Zhao, "Landmine detection with ground penetrating radar using hidden markov models," IEEE Trans. Geosci. Remote Sens., vol. 39, No. 6, pp. 1231-1244, June 2001.

[2] M. E. Yavuz, A. E. Fouda, and F. L. Teixeira, "GPR signal enhancement using sliding-window space-frequency matrices," Progress In Electromagnetics Research, vol. 145, pp. 1-10, 2014.

[3] P. Pratihar and A. K. Yadav, "Detection techniques for human safety from concealed weapon and harmful EDS," International Review of Applied Eng. Research., vol. 4, No. 1, pp. 71-76, 2014.

[4] W. Shao, B. Zhou, Z. Zheng, and G. Wang, "UWB microwave imaging for breast tumor detection in inhomogeneous tissue," Eng. in Med. and Biol. 27th Annual Conference, pp. 1496-1499, 2005.

[5] M. Fink, D. Cassereau, A. Derode, C. Prada, P. Roux, M. Tanter, J. Thomas, and F. Wu, "Time-reversed acoustics," Rep. Prog. Phys., vol. 63, pp. 1933-1995, 2000.

[6] C.-X. Li, W. Xu, J.-L. Li, and X.-Y. Gong, "Time-Reversal detection of multidimensional signals in underwater acoustics," IEEE J. Ocean. Eng., vol. 36, pp. 60-70, Jan 2011.

[7] R. Solimene, G. Leone, and A. Dell'Aversano, "MUSIC algorithms for rebar detection," J. Geophysics and Eng., vol. 10, No. 6, 2013.

[8] A. E. Fouda, F. L. Teixeira, and M. E. Yavuz, "Time-reversal techniques for MISO and MIMO wireless communication systems," Radio Science, vol. 47, 2012.

[9] J. M. F. Moura and Y. Jin, "Time reversal imaging by adaptive interference canceling," IEEE Trans. Signal Process., vol. 56, pp. 233-247, Jan 2008.

[10] J. M. F. Moura and Y. Jin, "Detection by time reversal: Single antenna," IEEE Trans. Signal Process., vol. 55, pp. 187-201, Jan 2007.

[11] M. E. Yavuz and F. L. Teixeira, "Frequency dispersion compensation in time reversal techniques for UWB electromagnetic waves," IEEE Geosci. Remote Sens. Lett., vol. 2, no. 2, pp. 233-237, April 2005.

[12] M. E. Yavuz and F. L. Teixeira, "Full time-domain DORT for ultrawideband electromagnetic fields in dispersive, random inhomogeneous media," IEEE Trans. Antennas Propag., vol. 54, pp. 2305-2315, Aug 2006.

[13] M. Tanter, J. L. Thomas, and M. Fink, "Focusing and steering through absorbing and aberrating layers: Application to ultrasonic propagation through the skull," J. Acoust. Soc. Am., vol. 103, pp. 2403-2410, May 1998.

[14] T. Folegot, C. Prada, and M. Fink, "Resolution enhancement and separation of reverberation from target echo with the time reversal operator decomposition," J. Acoust. Soc. Am., vol. 113, No. 6, pp. 31553160, 2003.

[15] M. Saillard, P. Vincent, and G. Micolau, "Reconstruction of buried objects surrounded by small inhomogeneities," Inverse Problems, vol. 16, pp. $1195-1208,2000$

[16] M. E. Yavuz and F. L. Teixeira, "Ultrawideband microwave sensing and imaging using time-reversal techniques: A review," Remote Sens., vol. 1, no. 3, pp. 466-495, 2009.

[17] A. V. Oppenheim, R. W. Schafer, and J. R. Buck., Discrete-Time Signal Processing. Upper Saddle River, NJ 07458: Prentice-Hall, Inc., 1999.

[18] F. Costen, J.-P. Bérenger, and A. Brown, "Comparison of FDTD hard source with FDTD soft source and accuracy assessment in Debye media," IEEE Trans. Antennas Propag., vol. 57, pp. 2014-2022, July 2009.
[19] J.-P. Bérenger, "Numerical reflection from FDTD-PMLs: A comparison of the split PML with the unsplit and CFS PMLs," IEEE Trans. Antennas Propag., vol. 50, No. 3, pp. 258-265, 2002.

[20] P. Debye, Polar Molecules. New York: Dover, 1929.

[21] RIKEN, Wako, Japan, "Media parameters for the Debye relaxation model." http://cfd-duo.riken.jp/cbms-mp/. [Online; accessed 26-April2016]. 